Pulsars : Problems \& Progress

ASP Conference Series, Vol. 105, 1996

S. Johnston, M. A. Walker and M. Bailes, eds.

\title{
Recent Results from the CANGAROO Project
}

\author{
S.A. Dazeley, P.G. Edwards, J.R. Patterson, G.P. Rowell, M. Sinnott, \\ G.J. Thornton, C. Wilkinson \\ CANGAROO Collaboration, Australia, \\ Department of Physics and Mathematical Physics, \\ University of Adelaide, 5005.
}

T. Hara, N. Hayashida, H. Katsumata, T. Kifune, T. Konishi, Y, Matsubara, T, Matsuoka, Y, Mizumoto, M. Mori, M, Muraishi;

Y. Muraki, T. Oda, S. Ogio, T. Ohsaki, T. Sako, K. Sakurazawa,

R. Susukita, A. Suzuki, T. Tamura, T. Tanimori, S. Yanagita,

T. Yoshida and T. Yoshikoshi

CANGAROO Collaboration, Japan, Institute for Cosmic Ray Research, University of Tokyo, Tokyo 188.

\section{The CANGAROO Project}

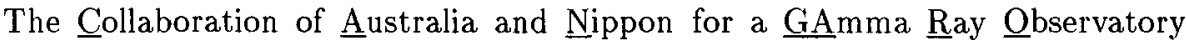
in the Qutback operates two large telescopes at Woomera (South Australia), which detect the Cerenkov light images produced in the atmosphere by electronpositron cascades initiated by very high energy $\left(\sim 1 \mathrm{TeV}\right.$ or $\left.10^{12} \mathrm{eV}\right)$ gamma rays. These gamma rays arise from a different mechanism than at EGRET energies: inverse Compton (IC) emission from relativistic electrons.

The spoke-like images are recorded by a multi-pixel camera which facilitates the rejection of the large numbers of oblique and ragged cosmic ray images. A field of view $\sim 3.5^{\circ}$ is required. The Australian team operates a triple $4 \mathrm{~m}$ diameter mirror telescope, BIGRAT, with a 37 photomultiplier tube camera and energy threshold $600 \mathrm{GeV}$. The Japanese operate a single, highly accurate $3.8 \mathrm{~m}$ diameter $\mathrm{f} / 1$ telescope and high resolution 256 photomultipler tube camera. In 1998 a new $7 \mathrm{~m}$ telescope is planned for Woomera with a design threshold $\sim 200 \mathrm{GeV}$.

\section{Egret pulsars}

\subsection{The Crab}

The Crab, a synchrotron nebula and pulsar at a distance of $1.5 \mathrm{kpc}$, is a standard candle for $\mathrm{TeV}$ work. We have observed it from Woomera, where it reaches a maximum elevation of $38^{\circ}$. At an energy threshold of $7 \mathrm{TeV}$, a $4 \sigma$ excess of gamma-ray events was found (Tanimori et al. 1994). More recent observations have confirmed this result. 


\subsection{PSR B1706-44}

This isolated southern $102 \mathrm{~ms}$ pulsar is only the second pulsar known to emit $\sim 1 \mathrm{TeV}$ photons so its confirmation and further study are important. Our observations were made in 1992-1993 and show steady non-periodic emission in both years with a combined statistical significance of $12 \sigma$ (Kifune et al. 1995). The measured flux of $1 \times 10^{-11}$ photons $\mathrm{cm}^{-2} \mathrm{~s}^{-1}$ above $1 \mathrm{TeV}$ is approximately 2 orders of magnitude below the extrapolated EGRET spectrum. Using BIGRAT data alone, the upper limit is $4.6 \times 10^{-11}$ photons $\mathrm{cm}^{-2} \mathrm{~s}^{-1}$ above $600 \mathrm{GeV}$.

\subsection{The Vela pulsar}

The Vela pulsar is young $(11,000 \mathrm{y})$ and relatively close $(\sim 500 \mathrm{pc})$. It is by far the brightest $\mathrm{GeV}$ gamma ray source observed by EGRET. The $\mathrm{GeV}$ emission is almost $100 \%$ pulsed. Recently Rosat observations below $2 \mathrm{keV}$ have revealed a relativistic jet extending SSW from the pulsar along the magnetic axis out to $0.4^{\circ}$ (Markwardt and Ogelman, 1995). emission. The TeV data show unpulsed gamma-ray emission from a position offset by $\sim 0.05^{\circ}$ from the pulsar, near the jet. An excess was detected each year over the three year period 1993-1995. The jet may cause shock acceleration of energetic particles which could result in the emission of $\mathrm{TeV}$ gamma rays. Further work is needed to confirm this important result.

\subsection{PSR B1055-52}

PSR $1055-52$ is a $197 \mathrm{~ms}$ EGRET pulsar at a distance of $1.5 \mathrm{kpc}$. It is notable as a very efficient converter of rotational energy into $\mathrm{GeV}$ gamma rays. However, no $\mathrm{TeV}$ emission has so far been observed in 2 years of CANGAROO observations.

\section{Other pulsars}

PSR $1259-63$ is a binary pulsar. At periastron in January 1994 , it passed very close to its companion. During these 4 days observation a $3 \sigma$ excess was observed by Cangaroo. Further observations are needed at the next periastron in 1997 .

PSR J0437-4715 is a binary $5.757 \mathrm{~ms}$ pulsar. The system is only 140 parsecs away. The spin down energy flux density, $\dot{E} / 4 \pi D^{2}$ is the same order of magnitude as PSR B1706-44. It has not been detected by EGRET. Our search for emission from PSR 0437-47 at the radio period yielded no evidence for $\mathrm{TeV}$ gamma ray emission. An upper limit for a pulsed flux above $\sim 1 \mathrm{TeV}$ of $5 \times 10^{-11}$ photons $\mathrm{cm}^{-2} \mathrm{~s}^{-1}$ was obtained by BIGRAT.

\section{References}

Kifune, T. et al., 1995, ApJ, 438, L91.

Markwardt, C.B. and Ogelman, H., 1995, Nature 375, 40.

Tanimori, T. et al., 1994, ApJ, 429, L61. 